\title{
On the stability of impulsive functional differential equations with infinite delays
}

\author{
Xiaodi Li,a, T. Caraballo, ${ }^{*,}$, R. Rakkiyappan ${ }^{\mathrm{c}}$, X. Han ${ }^{\mathrm{a}}$ \\ a School of Mathematical Sciences, Shandong Normal University, Ji'nan, 250014, P.R. China \\ ${ }^{b}$ Dpto. Ecuaciones Diferenciales y Análisis Numérico, Universidad de Sevilla, Apdo. de Correos 1160, \\ 41080-Sevilla, Spain \\ ${ }^{c}$ Department of Mathematics, Bharathiar University, Coimbatore - 641 046, Tamilnadu, India
}

\begin{abstract}
In this paper, the stability problem of impulsive functional differential equations with infinite delays is considered. By using Lyapunov functions and the Razumikhin technique, some new theorems on the uniform stability and uniform asymptotic stability are obtained. The obtained results are milder and more general than several recent works. Two examples are given to demonstrate the advantages of the results.
\end{abstract}

Key words: Infinite delays; Impulsive differential equations; Lyapunov functions; Stability

\section{Introduction}

As a popular and important topic, the stability problem of impulsive functional differential equations has generated a considerable interest in recent years, and a number of papers dealing with the stability problem of impulsive functional differential equations have appeared, see [1-12] and the references cited therein. In particular, stability of impulsive functional differential equations with infinite delays has recently received significant attention, see [13-21]. For example, Luo and Shen [13-15] studied the uniform asymptotic stability of impulsive functional differential equations with infinite delays by using Lyapunov functionals or/and Lyapunov functions and Razumikhin technique. In [16], Zhang and Sun extended the technique developed in [17] to impulsive systems and derived some new results on uniform stability of impulsive functional differential equations with infinite delays. Quite recently, Faria et al.[18] studied the existence and global stability for a class of non-autonomous impulsive functional

${ }^{*}$ Corresponding Author. Email address : caraball@us.es (T. Caraballo) 
differential equations with infinite delays via some analysis techniques. Our research group [19-21] also studied the stability problem of impulsive functional differential equations with infinite delays from impulsive perturbation and impulsive control point of view, respectively.

In the present paper, we will further investigate the stability problem of impulsive functional differential equations with infinite delays. By using Lyapunov functions and Razumikhin technique, some new results to guarantee uniform stability and the uniformly asymptotic stability are obtained. One of the most remarkable advantages of the results in this paper is that the Razumikhin condition is independent of impulses and enables one to deal with impulsive infinite delay differential equations with large impulsive perturbations. The methods developed in this paper extend and improve the results in [13-15,19,20]. Moreover, they can be applied to the some cases not covered by the results in $[9,11,12,16,18]$.

The paper is organized as follows. In Section 2, we introduce some definitions and notations. In Section 3, we present some new theorems on uniform stability and uniform asymptotic stability for impulsive functional differential equations with infinite delays. Two examples are given to illustrate the advantages of the results in Section 4. In Section 5, we draw a conclusion.

\section{Preliminaries}

Let $\mathbb{R}$ denote the set of real numbers, $\mathbb{R}_{+}$the set of positive real numbers and $\mathbb{R}^{n}$ the $n$ dimensional real space equipped with the Euclidean norm $|\bullet|$. Let $\mathbb{Z}_{+}$denote the set of positive integers, i.e., $\mathbb{Z}_{+}=\{1,2, \ldots\}$. For any interval $J \subseteq \mathbb{R}$, any subset $S \subseteq \mathbb{R}^{k}(1 \leq k \leq n), C(J, S)=$ $\{\varphi: J \rightarrow S$ is continuous $\}$ and $P C(J, S)=\{\varphi: J \rightarrow S$ is continuous everywhere except at finite number of points $t$, at which $\varphi\left(t^{+}\right), \varphi\left(t^{-}\right)$exist and $\left.\varphi\left(t^{+}\right)=\varphi(t)\right\}$. The impulse times $t_{k}$ satisfy $0 \leq t_{0}<t_{1}<\ldots<t_{k}<\ldots, \lim _{k \rightarrow+\infty} t_{k}=+\infty$. Denote by $\alpha$ a constant satisfying $-\infty \leq \alpha \leq 0$. In the case when $\alpha=-\infty$, the interval $[t+\alpha, t]$ is understood to be replaced by $(-\infty, t]$.

Consider the impulsive functional differential equations of the form

$$
\begin{cases}x^{\prime}(t)=f\left(t, x_{t}\right), & t \geq \sigma, t \neq t_{k}, \\ \left.\Delta x\right|_{t=t_{k}}=x\left(t_{k}\right)-x\left(t_{k}^{-}\right)=I_{k}\left(t_{k}, x\left(t_{k}^{-}\right)\right), & k \in \mathbb{Z}_{+}, \\ x_{\sigma}=\phi(s), & \alpha \leq s \leq 0,\end{cases}
$$


where $\sigma \geq t_{0}, \phi \in \mathbb{C}, f \in C\left(\left[t_{k-1}, t_{k}\right) \times \mathbb{C}, \mathbb{R}^{n}\right), f(t, 0)=0, \mathbb{C}$ is some open set in $P C\left([\alpha, 0], \mathbb{R}^{n}\right)$. Given a function $x(\cdot):[\alpha,+\infty) \rightarrow S$, for each $t \geq t_{0}$, we denote by $x_{t}$ is the element in $\mathbb{C}$ defined by $x_{t}(s)=x(t+s), s \in[\alpha, 0]$. Define $P C B=\{\varphi \in \mathbb{C}: \varphi$ is bounded $\}$ and for $\varphi \in P C B$, the norm of $\varphi$ is defined by $\|\varphi\|=\sup _{\alpha \leq \theta \leq 0}|\varphi(\theta)|$. Define $P C B_{\delta}=\{\varphi \in P C B:\|\varphi\| \leq \delta\}$. $I_{k}(t, x) \in C\left([0, \infty) \times \mathbb{R}^{n}, \mathbb{R}^{n}\right)$ and $I_{k}(t, 0)=0, k \in \mathbb{Z}_{+}$. Moreover, for any given $\rho>0$, there exists a $\rho_{1} \in(0, \rho)$ such that $x \in S\left(\rho_{1}\right)$ implies that $x+I_{k}\left(t_{k}, x\right) \in S(\rho)$, where $S(\rho)=\{x:|x|<$ $\left.\rho, x \in \mathbb{R}^{n}\right\}$.

In this paper, we assume that $f$ and $I_{k}$ satisfy certain conditions such that the solution of (1) exists on $[\sigma,+\infty)$ and is unique, see $[3,20]$ for detailed information. We denote by $x(t)=$ $x(t, \sigma, \phi)$ the solution of (1) with initial value $(\sigma, \phi)$. Since $f(t, 0)=0, I_{k}(t, 0)=0, k \in \mathbb{Z}_{+}$, then $x(t)=0$ is a solution of $(1)$, which is called the trivial solution.

We introduce some definitions (see [3]) as follows:

Definition 2.1. The function $V:[\alpha, \infty) \times \mathbb{C} \rightarrow \mathbb{R}_{+}$is said to belong to class $v_{0}$ if

(i) $V$ is continuous on each of the sets $\left[t_{k-1}, t_{k}\right) \times \mathbb{C}$ and $\lim _{(t, \varphi) \rightarrow\left(t_{k}^{-}, \psi\right)} V(t, \varphi)=V\left(t_{k}^{-}, \psi\right)$ exists;

(ii) $V(t, x)$ is locally Lipschitzian in $x$ and $V(t, 0) \equiv 0$.

Definition 2.2. Given a function $V \in v_{0}$, for any $(t, \psi) \in\left[t_{k-1}, t_{k}\right) \times \mathbb{C}$, the upper right-hand Dini derivative of $V$ along the solution of (1) is defined by

$$
D^{+} V(t, \psi(0))=\limsup _{h \rightarrow 0^{+}}\{V(t+h, \psi(0)+h f(t, \psi))-V(t, \psi(0))\} / h
$$

Definition 2.3. The trivial solution $x=0$ of (1) is said to be

$\left(P_{1}\right)$ stable, if for any $\sigma \geq t_{0}$ and $\varepsilon>0$, there exists a $\delta=\delta(\varepsilon, \sigma)>0$ such that $\phi \in P C B_{\delta}$ implies $|x(t, \sigma, \phi)|<\varepsilon, t \geq \sigma ;$

$\left(P_{2}\right)$ uniformly stable, if the $\delta$ in $\left(P_{1}\right)$ is independent on $\sigma$;

$\left(P_{3}\right)$ uniformly asymptotically stable, if $\left(P_{2}\right)$ holds and there exists some $\delta>0$ such that for any $\varepsilon>0$ there exists some $T=T(\varepsilon, \delta)>0$ such that $\phi \in P C B_{\delta}$ implies $|x(t, \sigma, \phi)|<$ $\varepsilon, t \geq \sigma+T$.

In addition, we define the following classes of functions for later use: 
$K_{1}=\left\{a \in C\left(\mathbb{R}_{+}, \mathbb{R}_{+}\right) \mid a(0)=0\right.$ and $a(s)>0$ for $\left.s>0\right\}$

$K_{2}=\left\{a \in C\left(\mathbb{R}_{+}, \mathbb{R}_{+}\right) \mid a \in K_{1}\right.$ and $a$ is non-decreasing in $\left.s\right\}$.

\section{Stability results}

Now we can state our main stability result.

Theorem 3.1. Assume that there exist some functions $W_{1}, W_{2} \in K_{2}, P, G \in K_{1}, q \in C\left(\mathbb{R}_{+}, \mathbb{R}_{+}\right)$, $g \in P C\left(\mathbb{R}_{+}, \mathbb{R}_{+}\right), V(t, x) \in v_{0}$ and constants $\beta_{k} \geq 0, k \in \mathbb{Z}_{+}$such that

(i) $W_{1}(|x|) \leq V(t, x) \leq W_{2}(|x|),(t, x) \in\left[t_{0}, \infty\right) \times S(\rho)$;

(ii) For any $\left(t_{k}, \psi\right) \in \mathbb{R}_{+} \times P C\left([\alpha, 0], S\left(\rho_{1}\right)\right)$,

$$
V\left(t_{k}, \psi(0)+I_{k}\left(t_{k}, \psi\right)\right)-V\left(t_{k}^{-}, \psi(0)\right) \leq \beta_{k} V\left(t_{k}^{-}, \psi(0)\right),
$$

where $\sum_{k=1}^{\infty} \beta_{k} \doteq \beta<\infty$;

(iii) For any $\sigma \geq t_{0}$ and $\psi \in P C([\alpha, 0], S(\rho))$, if $P(V(t, \psi(0)))>V(t+\theta, \psi(\theta))$ for $\max \{\alpha,-q(V(t))\} \leq$ $\theta \leq 0$, then

$$
D^{+} V(t, \psi(0)) \leq-g(t) G(V(t, \psi(0))), t \in\left[t_{k-1}, t_{k}\right), k \in \mathbb{Z}_{+},
$$

where $P(s)>$ for $s>0$;

(iv) For any given $\varepsilon_{2}>\varepsilon_{1}>0$, there exists a $\eta=\eta\left(\varepsilon_{1}, \varepsilon_{2}\right)>0$ such that for any $A>0$ implies that

$$
\int_{A}^{A+\eta} g(t) d t>\frac{(1+\beta) W_{2}\left(\varepsilon_{2}\right)}{M}
$$

where $M=\inf _{0.5 W_{1}\left(\varepsilon_{1}\right) \leq s \leq W_{2}\left(\varepsilon_{2}\right)} G(s)$.

Then the trivial solution of (1) is uniformly asymptotically stable.

Proof. We first show that the trivial solution of (1) is uniformly stable.

For any $\varepsilon \in\left(0, \rho_{1}\right)$, one may choose $\delta>0$ such that $W_{2}(\delta) \leq \beta^{\star-1} W_{1}(\varepsilon)$, where $\beta^{\star} \doteq$ $\prod_{k=1}^{\infty}\left(1+\beta_{k}\right)+1$. For any $\sigma \geq t_{0}$ and $\phi \in P C B_{\delta}$, let $x(t)=x(t, \sigma, \phi)$ be a solution of (1) through $(\sigma, \phi)$.

Note that $\phi \in P C B_{\delta}$, it is obvious that

$$
W_{1}(|x|) \leq V(t, x(t)) \leq W_{2}(\delta) \leq \beta^{\star-1} W_{1}(\varepsilon)<W_{1}(\varepsilon), \sigma+\alpha \leq t \leq \sigma,
$$


which implies that $|x(t)|<\rho_{1}, t \in[\sigma+\alpha, \sigma]$.

Suppose that $\sigma \in\left[t_{m-1}, t_{m}\right)$ for some $m \in \mathbb{Z}_{+}$, then we can prove for $t \in\left[\sigma, t_{m}\right)$

$$
V(t, x(t)) \leq \beta^{\star-1} W_{1}(\varepsilon)
$$

If this is not true, then there exists some $t \in\left[\sigma, t_{m}\right)$ such that $V(t, x(t))>\beta^{\star-1} W_{1}(\varepsilon)$.

Define

$$
t^{\star}=\inf \left\{t \in\left[\sigma, t_{m}\right), V(t, x(t))>\beta^{\star-1} W_{1}(\varepsilon)\right\},
$$

then it is obvious that $t^{\star} \geq \sigma, V\left(t^{\star}, x\left(t^{\star}\right)\right)=\beta^{\star-1} W_{1}(\varepsilon)$ and $V(t, x(t)) \leq \beta^{\star-1} W_{1}(\varepsilon), t \in\left[\sigma, t^{\star}\right]$.

Meanwhile, we know

$$
\left.D^{+} V\right|_{(1)}\left(t^{\star}, x\left(t^{\star}\right)\right) \geq 0 \text {. }
$$

In this case, it holds

$$
P\left(V\left(t^{\star}, x\left(t^{\star}\right)\right)\right)>V\left(t^{\star}, x\left(t^{\star}\right)\right)=\beta^{\star-1} W_{1}(\varepsilon) \geq V(s, x(s)), t^{\star}+\alpha \leq s \leq t^{\star} .
$$

By condition ( $i i i), g \in P C\left(\mathbb{R}_{+}, \mathbb{R}_{+}\right)$, and $G \in K_{1}$, we obtain

$$
D^{+} V\left(t^{\star}, x\left(t^{\star}\right)\right) \leq-g\left(t^{\star}\right) G\left(V\left(t^{\star}, x\left(t^{\star}\right)\right)\right)=-g\left(t^{\star}\right) G\left(\beta^{\star-1} W_{1}(\varepsilon)\right)<0,
$$

which is a contradiction with (3). Thus (2) holds. It implies that $x\left(t_{m}^{-}\right) \in S\left(\rho_{1}\right), x\left(t_{m}\right) \in S(\rho)$. Then note that

$$
V\left(t_{m}, x\left(t_{m}\right)\right) \leq\left(1+\beta_{m}\right) V\left(t_{m}^{-}, x\left(t_{m}^{-}\right)\right) \leq \beta^{\star-1}\left(1+\beta_{m}\right) W_{1}(\varepsilon) .
$$

We next can prove that for $t \in\left[t_{m}, t_{m+1}\right)$

$$
V(t, x(t)) \leq \beta^{\star-1}\left(1+\beta_{m}\right) W_{1}(\varepsilon) .
$$

Suppose that this is not true, then we can define

$$
t^{*}=\inf \left\{t \in\left[t_{m}, t_{m+1}\right), V(t, x(t))>\beta^{\star-1}\left(1+\beta_{m}\right) W_{1}(\varepsilon)\right\} .
$$

Thus, we can obtain a contradiction by the same arguments as the proof of (2), and we will therefore omit the details.

By induction hypothesis, we may prove that for $t \in\left[\sigma, t_{m}\right) \cup\left[t_{k}, t_{k+1}\right), k \geq m$,

$$
V(t, x(t)) \leq \beta^{\star-1}\left(1+\beta_{m}\right)\left(1+\beta_{m+1}\right) \cdots\left(1+\beta_{k}\right) W_{1}(\varepsilon),
$$


which yields

$$
W_{1}(\|x\|) \leq V(t, x(t)) \leq \beta^{\star^{-1}} \prod_{\sigma<t_{k}<t}\left(1+\beta_{k}\right) W_{1}(\varepsilon)<W_{1}(\varepsilon), t \geq \sigma .
$$

Hence, $|x(t)|<\varepsilon, t \geq \sigma$. In view of the choice of $\delta$, the trivial solution of (1) is uniformly stable.

Next we show the uniformly asymptotic stability.

Since the trivial solution of (1) is uniformly stable, for any given $\varepsilon_{2} \in\left(0, \rho_{1}\right), \sigma \geq t_{0}$, we can find a corresponding $\delta=\delta\left(\varepsilon_{2}\right)>0$ such that for any $\phi \in P C B_{\delta}$ implies that $|x(t)| \leq \varepsilon_{2}<$ $\rho_{1}, t \geq \sigma$ and $V(t, x(t)) \leq W_{2}\left(\varepsilon_{2}\right), t \geq \sigma$. In the sequel, we assume without loss of generality that $\sigma \in\left[t_{m_{1}-1}, t_{m_{1}}\right), m_{1} \in \mathbb{Z}_{+}$.

For any $\varepsilon \in\left(0, \varepsilon_{2}\right)$, choose constants $M$ and $a$ as follows:

$$
\begin{aligned}
& M=M\left(\varepsilon_{2}, \varepsilon\right)=\inf _{0.5 W_{1}(\varepsilon) \leq s \leq W_{2}\left(\varepsilon_{2}\right)} G(s), \\
& a=a\left(\varepsilon_{2}, \varepsilon\right)=\min \left\{\inf _{0.5 W_{1}(\varepsilon) \leq s \leq W_{2}\left(\varepsilon_{2}\right)}[P(s)-s], 0.5 W_{1}(\varepsilon)\right\} .
\end{aligned}
$$

Then it is obvious that $M>0, a>0$. Also, from condition (iv), we know that there exists $\eta=\eta\left(\varepsilon, \varepsilon_{2}\right)>0$ such that for any $A>0$ implies that

$$
\int_{A}^{A+\eta} g(t) d t>\frac{(1+\beta) W_{2}\left(\varepsilon_{2}\right)}{M} .
$$

Now we choose $N \in \mathbb{Z}_{+}$such that

$$
0.5 W_{1}(\varepsilon)+(N-1) a \leq W_{2}\left(\varepsilon_{2}\right)<0.5 W_{1}(\varepsilon)+N a .
$$

Since $\sum_{k=1}^{\infty} \beta_{k}<\infty$, there exists a large enough integer $N_{0}>m_{1}$ such that

$$
\sum_{i=N_{0}}^{\infty} \beta_{i}<\frac{a}{3 W_{2}\left(\varepsilon_{2}\right)} \text {, and } \beta_{k}<\frac{a}{3 N W_{1}(\varepsilon)}, k \geq N_{0} .
$$

Suppose that $t_{N_{0}}=\sigma+\lambda \eta$, where $\lambda$ is a constant. Then we show that there exists $T_{1}>t_{N_{0}}$ such that

$$
V\left(T_{1}, x\left(T_{1}\right)\right)<0.5 W_{1}(\varepsilon)+(N-1) a .
$$

Suppose on the contrary, then for all $t>t_{N_{0}}$

$$
V(t, x(t)) \geq 0.5 W_{1}(\varepsilon)+(N-1) a \geq 0.5 W_{1}(\varepsilon) .
$$


In view of the definition of $a$, we have

$$
\begin{aligned}
P(V(t, x(t))) & \geq V(t, x(t))+a \\
& \geq 0.5 W_{1}(\varepsilon)+(N-1) a+a \\
& =0.5 W_{1}(\varepsilon)+N a \\
& >W_{2}\left(\varepsilon_{2}\right) \geq V(s, x(s)), t+\alpha \leq s \leq t, t>t_{N_{0}} .
\end{aligned}
$$

By assumption (iii), we obtain that the inequality $D^{+} V(t, x(t)) \leq-g(t) G(V(t, x(t)))$ holds for all $t>t_{N_{0}}, t \neq t_{k}$. Integrating above inequality from $t_{N_{0}}$ to $t_{N_{0}}+\eta$, by (4) we get

$$
\begin{aligned}
V\left(t_{N_{0}}+\eta, x\left(t_{N_{0}}+\eta\right)\right) \leq & V\left(t_{N_{0}}, x\left(t_{N_{0}}\right)\right)-\int_{t_{N_{0}}}^{t_{N_{0}}+\eta} g(s) G(V(s)) d s \\
& +\sum_{t_{N_{0}}<t<t_{N_{0}}+\eta}\left[V\left(t_{k}\right)-V\left(t_{k}^{-}\right)\right] \\
\leq & V\left(t_{N_{0}}, x\left(t_{N_{0}}\right)\right)-M \int_{t_{N_{0}}}^{t_{N_{0}}+\eta} g(s) d s \\
& +\sum_{t_{N_{0}}<t<t_{N_{0}}+\eta} \beta_{k} V\left(t_{k}^{-}\right) \\
\leq & W_{2}\left(\varepsilon_{2}\right)-M \int_{t_{N_{0}}}^{t_{N_{0}}+\eta} g(s) d s \\
& +\sum_{t_{N_{0}}<t<t_{N_{0}}+\eta} \beta_{k} W_{2}\left(\varepsilon_{2}\right) \\
\leq & W_{2}\left(\varepsilon_{2}\right)(1+\beta)-M \int_{t_{N_{0}}}^{t_{N_{0}}+\eta} g(s) d s \\
< & 0,
\end{aligned}
$$

which is a contradiction. Thus (6) holds. One may choose $T_{1}=t_{N_{0}}+\eta=\sigma+(\lambda+1) \eta$.

We next show that for all $t>T_{1}$

$$
V(t, x(t))<0.5 W_{1}(\varepsilon)+(N-1) a+\frac{a}{2} .
$$

Suppose this is not true, then there exists $\tau_{2}>T_{1}$ such that

$$
V\left(\tau_{2}, x\left(\tau_{2}\right)\right) \geq 0.5 W_{1}(\varepsilon)+(N-1) a+\frac{a}{2}
$$

and

$$
V(t, x(t))<0.5 W_{1}(\varepsilon)+(N-1) a+\frac{a}{2} \text { for all } T_{1} \leq t<\tau_{2} .
$$


Suppose that $T_{1} \in\left[t_{m}, t_{m+1}\right), m \geq N_{0}, m \in \mathbb{Z}_{+}$, then we claim that $\tau_{2} \geq t_{m+1}$. Otherwise, then $\tau_{2} \in\left[T_{1}, t_{m+1}\right)$. Since (6) holds, it is clear that there exists $\tau_{1} \in\left[T_{1}, \tau_{2}\right)$ such that

$$
V\left(\tau_{1}, x\left(\tau_{1}\right)\right)=0.5 W_{1}(\varepsilon)+(N-1) a
$$

and

$$
V\left(\tau_{1}, x\left(\tau_{1}\right)\right) \leq V(t, x(t)) \leq V\left(\tau_{2}, x\left(\tau_{2}\right)\right), \tau_{1} \leq t \leq \tau_{2}
$$

Then we have for $t \in\left[\tau_{1}, \tau_{2}\right]$

$$
\begin{aligned}
P(V(t, x(t))) & \geq V(t, x(t))+a \\
& \geq 0.5 W_{1}(\varepsilon)+N a \\
& >W_{2}\left(\varepsilon_{2}\right) \geq V(s, x(s)), t+\alpha \leq s \leq t .
\end{aligned}
$$

Using condition (iii), we have

$$
D^{+} V(t, x(t)) \leq-g(t) G(V(t)) \leq 0, \tau_{1} \leq t \leq \tau_{2},
$$

which implies

$$
V\left(\tau_{2}, x\left(\tau_{2}\right)\right) \leq V\left(\tau_{1}, x\left(\tau_{1}\right)\right)
$$

This is a contradiction in view of (8). Then we have proven that $\tau_{2} \geq t_{m+1}$. Without loss of generality, we may suppose that $\tau_{2} \in\left[t_{m+q}, t_{m+q+1}\right), q \geq 1$. Next we shall claim that there exists $\tau_{1}^{\prime} \in\left(T_{1}, \tau_{2}\right)$ such that

$$
0.5 W_{1}(\varepsilon)+(N-1) a<V\left(\tau_{1}^{\prime}, x\left(\tau_{1}^{\prime}\right)\right)<0.5 W_{1}(\varepsilon)+(N-1) a+\frac{a}{2} .
$$

By virtue of (9), we only need to prove the left-hand inequality of (10). Suppose this inequality does not hold, then for all $t \in\left(T_{1}, \tau_{2}\right)$,

$$
V(t, x(t)) \leq 0.5 W_{1}(\varepsilon)+(N-1) a .
$$

Then by (8), we know that there must be $\tau_{2}=t_{m+q}$. It follows that

$$
V\left(t_{m+q}, x\left(t_{m+q}\right)\right) \geq 0.5 W_{1}(\varepsilon)+(N-1) a+\frac{a}{2}, V\left(t_{m+q}^{-}, x\left(t_{m+q}^{-}\right)\right) \leq 0.5 W_{1}(\varepsilon)+(N-1) a,
$$

which together with condition (ii) yields

$$
\frac{a}{2} \leq \beta_{m+q} V\left(t_{m+q}^{-}, x\left(t_{m+q}^{-}\right)\right) \leq \beta_{m+q} W_{2}\left(\varepsilon_{2}\right) .
$$


Thus, it leads to

$$
\beta_{m+q} \geq \frac{a}{2 W_{2}\left(\varepsilon_{2}\right)} .
$$

This contradicts the first inequality of (5) and thus (10) holds.

Defining now

$$
\widetilde{\tau_{1}}=\sup \left\{t \in\left[T_{1}, \tau_{2}\right], V(t, x(t))<0.5 W_{1}(\varepsilon)+(N-1) a\right\}
$$

then

$$
\begin{aligned}
& V\left(\widetilde{\tau_{1}}, x\left(\widetilde{\tau_{1}}\right)\right) \leq 0.5 W_{1}(\varepsilon)+(N-1) a, \\
& V\left(\widetilde{\tau_{1}}, x\left(\widetilde{\tau_{1}}\right)\right)=V\left({\widetilde{\tau_{1}}}^{+}, x\left({\widetilde{\tau_{1}}}^{+}\right)\right) \geq 0.5 W_{1}(\varepsilon)+(N-1) a
\end{aligned}
$$

and

$$
0.5 W_{1}(\varepsilon)+(N-1) a \leq V(t, x(t)) \leq 0.5 W_{1}(\varepsilon)+(N-1) a+\frac{a}{2}, t \in\left[\widetilde{\tau_{1}}, \tau_{2}\right] .
$$

By virtue of (10), we know that $\widetilde{\tau_{1}}<\tau_{2}$. Note that $\tau_{2} \in\left[t_{m+q}, t_{m+q+1}\right)$, we further show that $\widetilde{\tau_{1}}<t_{m+q}$. Suppose on the contrary that $\widetilde{\tau_{1}} \in\left[t_{m+q}, \tau_{2}\right)$, then there is no impulse point $t_{k}$ between $\widetilde{\tau_{1}}$ and $\tau_{2}$.

From (12), we have

$$
\begin{aligned}
P(V(t, x(t))) & \geq V(t, x(t))+a \\
& =0.5 W_{1}(\varepsilon)+N a \\
& >W_{2}\left(\varepsilon_{2}\right) \geq V(s, x(s)), t+\alpha \leq s \leq t, \widetilde{\tau_{1}} \leq t \leq \tau_{2} .
\end{aligned}
$$

By assumption (iii), we obtain

$$
D^{+} V(t, x(t)) \leq-g(t) G(V(t)) \leq 0, \quad \widetilde{\tau_{1}} \leq t \leq \tau_{2},
$$

which implies that

$$
V\left(\tau_{2}, x\left(\tau_{2}\right)\right) \leq V\left(\widetilde{\tau_{1}}, x\left(\widetilde{\tau_{1}}\right)\right)
$$

This is a contradiction with the definition of $\widetilde{\tau_{1}}$. Consequently, we have that $\widetilde{\tau_{1}}<t_{m+q}$.

Suppose that $\widetilde{\tau_{1}} \in\left[t_{m+k}, t_{m+k+1}\right), 1 \leq k<q$, then we now consider the following two possible cases:

Case 1: If $\widetilde{\tau_{1}}>t_{m+k}$, i.e., $\widetilde{\tau_{1}} \in\left(t_{m+k}, t_{m+k+1}\right)$, then considering the definition of $\widetilde{\tau_{1}}$, we have

$$
V\left(\widetilde{\tau_{1}}, x\left(\widetilde{\tau_{1}}\right)\right)=0.5 W_{1}(\varepsilon)+(N-1) a .
$$


From (12), we can deduce that, for $t \in\left[\widetilde{\tau_{1}}, \tau_{2}\right]$,

$$
P(V(t, x(t))) \geq V(t, x(t))+a>V(s, x(s)), \quad t+\alpha \leq s \leq t .
$$

By (iii), the inequality $D^{+} V(t, x(t)) \leq-g(t) G(V(t)) \leq 0$ holds for $t \in\left[\widetilde{\tau_{1}}, \tau_{2}\right]$. Thus we arrive at

$$
\begin{aligned}
0.5 W_{1}(\varepsilon)+(N-1) a+\frac{a}{2} & \leq V\left(\tau_{2}, x\left(\tau_{2}\right)\right) \\
& \leq V\left(\widetilde{\tau_{1}}, x\left(\widetilde{\tau_{1}}\right)\right)+\sum_{i=m+k+1}^{m+q}\left[V\left(t_{i}\right)-V\left(t_{i}^{-}\right)\right] \\
& \leq 0.5 W_{1}(\varepsilon)+(N-1) a+\sum_{i=m+k+1}^{m+q} \beta_{i} V\left(t_{i}^{-}\right) \\
& \leq 0.5 W_{1}(\varepsilon)+(N-1) a+\sum_{i=m+k+1}^{m+q} \beta_{i} W_{2}\left(\varepsilon_{2}\right),
\end{aligned}
$$

which yields

$$
\frac{a}{2} \leq \sum_{i=m+k+1}^{m+q} \beta_{i} W_{2}\left(\varepsilon_{2}\right) .
$$

This is a contradiction with the first inequality of (5). Hence, Case 1 could not happen.

Case 2: If $\widetilde{\tau_{1}}=t_{m+k}$, then by (11), we know

$$
V\left(t_{m+k}^{-}, x\left(t_{m+k}^{-}\right)\right) \leq 0.5 W_{1}(\varepsilon)+(N-1) a .
$$

Therefore,

$$
\begin{aligned}
V\left(\widetilde{\tau_{1}}, x\left(\widetilde{\tau_{1}}\right)\right)=V\left(t_{m+k}, x\left(t_{m+k}\right)\right) & \leq\left(1+\beta_{m+k}\right) V\left(t_{m+k}^{-}, x\left(t_{m+k}^{-}\right)\right) \\
& \leq\left(1+\beta_{m+k}\right)\left[0.5 W_{1}(\varepsilon)+(N-1) a\right] .
\end{aligned}
$$

From (12), it still holds that $P(V(t, x(t)))>V(s, x(s)), t+\alpha \leq s \leq t, \widetilde{\tau_{1}} \leq t \leq \tau_{2}$. Using assumption (iii) again, we obtain that the inequality $D^{+} V(t, x(t)) \leq-g(t) G(V(t)) \leq 0$ holds for $t \in\left[\widetilde{\tau_{1}}, \tau_{2}\right]$. Hence, in this case we derive

$$
\begin{aligned}
0.5 W_{1}(\varepsilon)+(N-1) a+\frac{a}{2} \leq & V\left(\tau_{2}, x\left(\tau_{2}\right)\right) \\
\leq & V\left(\widetilde{\tau_{1}}, x\left(\widetilde{\tau_{1}}\right)\right)+\sum_{i=m+k+1}^{m+q}\left[V\left(t_{i}\right)-V\left(t_{i}^{-}\right)\right] \\
< & \left(1+\beta_{m+k}\right)\left[0.5 W_{1}(\varepsilon)+(N-1) a\right] \\
& +\sum_{i=m+k+1}^{m+q} \beta_{i} V\left(t_{i}^{-}\right),
\end{aligned}
$$


which, together with the latter inequality of (5) and the fact that $a \leq 0.5 W_{1}(\varepsilon)$ yields

$$
\begin{aligned}
\frac{a}{2} & \leq \beta_{m+k}\left[0.5 W_{1}(\varepsilon)+(N-1) a\right]+\sum_{i=m+k+1}^{m+q} \beta_{i} W_{2}\left(\varepsilon_{2}\right) \\
& \leq \beta_{m+k} N 0.5 W_{1}(\varepsilon)+\sum_{i=m+k+1}^{m+q} \beta_{i} W_{2}\left(\varepsilon_{2}\right) \\
& \leq \frac{a}{3 N W_{1}(\varepsilon)} \cdot N 0.5 W_{1}(\varepsilon)+\sum_{i=m+k+1}^{m+q} \beta_{i} W_{2}\left(\varepsilon_{2}\right) .
\end{aligned}
$$

That is,

$$
\frac{a}{3} \leq \sum_{i=m+k+1}^{m+q} \beta_{i} W_{2}\left(\varepsilon_{2}\right)
$$

which is a contradiction with (5). Therefore, Case 2 could not happen either. Therefore, we have proven that (7) holds for all $t>T_{1}$.

By now, we have the following assertion by (6) and (7):

$$
\left\{\begin{array}{l}
V\left(T_{1}, x\left(T_{1}\right)\right)<0.5 W_{1}(\varepsilon)+(N-1) a, \\
V(t, x(t))<0.5 W_{1}(\varepsilon)+(N-1) a+\frac{a}{2}, t>T_{1},
\end{array}\right.
$$

where $T_{1}=\sigma+(\lambda+1) \eta$.

Define a constant $q$ as follows:

$$
q=\sup \left\{q(s) \mid 0.5 W_{1}(\varepsilon) \leq s \leq W_{2}\left(\varepsilon_{2}\right)\right\} .
$$

Then it can be deduced that there exists $T_{2}>T_{1}+q$ such that

$$
V\left(T_{2}, x\left(T_{2}\right)\right)<0.5 W_{1}(\varepsilon)+(N-2) a+\frac{a}{2},
$$

whose proof is similar to the proof of (6) under the help of (13), and we only need to note the following Razumikhin condition :

$$
\begin{aligned}
P(V(t, x(t))) & \geq V(t, x(t))+a \\
& \geq 0.5 W_{1}(\varepsilon)+(N-1) a+\frac{a}{2} \\
& >V(s, x(s)), \max \{t+\alpha, t-q(V(t))\} \leq s \leq t, t>T_{1}+q .
\end{aligned}
$$

Choose $T_{2}=T_{1}+q+\eta=\sigma+(\lambda+1) \eta+q$. Then applying the same argument as (7), we can show that for all $t>T_{2}$

$$
V(t, x(t))<0.5 W_{1}(\varepsilon)+(N-1) a, t>T_{2} .
$$


In this way, we can prove that for $j \in \mathbb{Z}_{+}$,

$$
\left\{\begin{array}{l}
V\left(T_{j}, x\left(T_{j}\right)\right)<0.5 W_{1}(\varepsilon)+(N-1) a-\frac{j-1}{2} a, \\
V(t, x(t))<0.5 W_{1}(\varepsilon)+(N-1) a-\frac{j-2}{2} a, t>T_{j},
\end{array}\right.
$$

where $T_{j}=\sigma+(\lambda+1) \eta+(q+\eta)(j-1)$. In particular, let $j=2 N$, then we obtain that $V(t, x(t))<$ $0.5 W_{1}(\varepsilon)<W_{1}(\varepsilon), t>T_{2 N}$. It implies that $|x(t)|<\varepsilon, t>T_{2 N}$. Note that $(\lambda+1) \eta+(q+\eta)(2 N-1)$ is independent of $\sigma$, then we obtain that the trivial solution of (1) is uniformly asymptotically stable. The proof of Theorem 3.1 is therefore complete.

If we only consider the uniform stability of (1), then the following result can be obtained.

Corollary 3.1. The trivial solution of (1) is uniformly stable if there exist some functions $W_{1}, W_{2} \in K_{2}, P, G \in K_{1}, g \in P C\left(\mathbb{R}_{+}, \mathbb{R}_{+}\right), V(t, x) \in v_{0}$ and constants $\beta_{k} \geq 0, k \in \mathbb{Z}_{+}$such that conditions (i),(ii) (iv) in Theorem 3.1 and (v) hold, where

(v) For any $\sigma \geq t_{0}$ and $\psi \in P C([\alpha, 0], S(\rho))$, if $P(V(t, \psi(0)))>V(t+\theta, \psi(\theta))$ for $\alpha \leq \theta \leq 0$, then

$$
D^{+} V(t, \psi(0)) \leq-g(t) G(V(t, \psi(0))), t \in\left[t_{k-1}, t_{k}\right), k \in \mathbb{Z}_{+},
$$

where $P(s)>$ for $s>0$.

On the other hand, if function $g(t)$ satisfies $\inf _{t \in \mathbb{R}_{+}} g(t) \doteq \mu>0$, then by Theorem 3.1 and Corollary 3.1, we have the following results, respectively.

Corollary 3.2. The trivial solution of (1) is uniformly asymptotically stable if there exist some functions $W_{1}, W_{2} \in K_{2}, P, G \in K_{1}, V(t, x) \in v_{0}$ and constants $\mu>0, \beta_{k} \geq 0, k \in \mathbb{Z}_{+}$such that conditions (i),(ii) in Theorem 3.1 and (vi) hold, where

(vi) For any $\sigma \geq t_{0}$ and $\psi \in P C([\alpha, 0], S(\rho))$, if $P(V(t, \psi(0)))>V(t+\theta, \psi(\theta))$ for $\max \{\alpha,-q(V(t))\} \leq$ $\theta \leq 0$, then

$$
D^{+} V(t, \psi(0)) \leq-\mu G(V(t, \psi(0))), t \in\left[t_{k-1}, t_{k}\right), k \in \mathbb{Z}_{+},
$$

where $P(s)>s$ for $s>0$.

Corollary 3.3. The trivial solution of (1) is uniformly stable if there exist some functions $W_{1}, W_{2} \in K_{2}, P, G \in K_{1}, V(t, x) \in v_{0}$ and constants $\mu>0, \beta_{k} \geq 0, k \in \mathbb{Z}_{+}$such that conditions (i),(ii) in Theorem 3.1 and (vii) hold, where 
(vii) For any $\sigma \geq t_{0}$ and $\psi \in P C([\alpha, 0], S(\rho))$, if $P(V(t, \psi(0)))>V(t+\theta, \psi(\theta))$ for $\alpha \leq \theta \leq 0$, then

$$
D^{+} V(t, \psi(0)) \leq-\mu G(V(t, \psi(0))), t \in\left[t_{k-1}, t_{k}\right), k \in \mathbb{Z}_{+},
$$

where $P(s)>s$ for $s>0$.

Proof. For any given $\varepsilon_{2}>\varepsilon_{1}>0$, one can choose $\eta=\frac{(1+\beta) W_{2}\left(\varepsilon_{2}\right)}{\mu M}$, where $M=\inf _{0.5 W_{1}\left(\varepsilon_{1}\right) \leq s \leq W_{2}\left(\varepsilon_{2}\right)} G(s)$. Then, we can obtain the above results easily.

\section{Examples}

In this section, we present two examples to illustrate our results.

Example 4.1. Consider the impulsive functional differential equations with infinite delay (see [16])

$$
\begin{cases}x_{1}^{\prime}(t)=-a_{1} x_{1}(t)+a_{2} x_{2}(t)+a_{3} x_{1}(t-\tau(t)), & t \geq t_{0}, t \neq t_{k}, \\ x_{2}^{\prime}(t)=b_{1} x_{1}(t)-b_{2} x_{2}(t)+b_{3} x_{2}(t-\tau(t)), & t \geq t_{0}, t \neq t_{k}, \\ x_{1}\left(t_{k}\right)=\beta_{k} x_{1}\left(t_{k}^{-}\right), & k \in \mathbb{Z}_{+}, \\ x_{2}\left(t_{k}\right)=\gamma_{k} x_{2}\left(t_{k}^{-}\right), & k \in \mathbb{Z}_{+},\end{cases}
$$

where $0 \leq \tau(t) \leq t, a_{j}>0, b_{j}>0, j=1,2,3$, the impulse points $t_{k}$ satisfy $0 \leq t_{0}<$ $t_{1}<\ldots<t_{k}<\ldots, \lim _{k \rightarrow \infty} t_{k}=\infty, \beta_{k}, \gamma_{k}, k \in \mathbb{Z}_{+}$are some positive constants which satisfy $\prod_{k=1}^{\infty} \max \left\{\beta_{k}, \gamma_{k}\right\}<\infty$.

Property 4.1. Assume that the following condition holds:

$$
\max \left\{a_{3}, b_{3}\right\}<\min \left\{2 a_{1}-a_{2}-a_{3}-b_{1}, 2 b_{2}-a_{2}-b_{3}-b_{1}\right\}
$$

then the zero solution of (14) is uniformly asymptotically stable.

Proof. Since (15) holds, one may choose $P(s)=\lambda s$, where

$$
\lambda=\frac{\min \left\{2 a_{1}-a_{2}-a_{3}-b_{1}, 2 b_{2}-a_{2}-b_{3}-b_{1}\right\}}{2 \max \left\{a_{3}, b_{3}\right\}}+\frac{1}{2} .
$$

Then it is obvious that $P(s)>s$ for $s>0$.

Let $V(t, x)=x_{1}^{2}(t)+x_{2}^{2}(t), W_{1}(s)=W_{2}(s)=s^{2}$, then condition $(i)$ in Theorem 3.1 holds. In addition, when $P(V(t, \psi(0)))>V(t+\theta, \psi(\theta)), \alpha \leq \theta \leq 0$, i.e., $\lambda\left(x_{1}^{2}(t)+x_{2}^{2}(t)\right)>x_{1}^{2}(s)+x_{2}^{2}(s), t+$ 
$\alpha \leq s \leq t$, we have

$$
\begin{aligned}
& \left.D^{+} V\right|_{(14)}=2 x_{1}(t)\left\{-a_{1} x_{1}(t)+a_{2} x_{2}(t)+a_{3} x_{1}(t-\tau(t))\right\}+2 x_{2}\left\{b_{1} x_{1}(t)\right. \\
& \left.-b_{2} x_{2}(t)+b_{3} x_{2}(t-\tau(t))\right\} \\
& \leq-2 a_{1} x_{1}^{2}(t)+2 a_{2} x_{1}(t) x_{2}(t)+2 a_{3} x_{1}(t) x_{1}(t-\tau(t)) \\
& +2 b_{1} x_{1}(t) x_{2}(t)-2 b_{2} x_{2}^{2}(t)+2 b_{3} x_{2}(t) x_{2}(t-\tau(t)) \\
& \leq-2 a_{1} x_{1}^{2}(t)+a_{2}\left(x_{1}^{2}(t)+x_{2}^{2}(t)\right)+a_{3}\left(x_{1}^{2}(t)+x_{1}^{2}(t-\tau(t))\right) \\
& -2 b_{2} x_{2}^{2}(t)+b_{1}\left(x_{1}^{2}(t)+x_{2}^{2}(t)\right)+b_{3}\left(x_{2}^{2}(t)+x_{2}^{2}(t-\tau(t))\right) \\
& \leq\left(-2 a_{1}+a_{2}+a_{3}+b_{1}\right) x_{1}^{2}(t)+\left(-2 b_{2}+a_{2}+b_{3}+b_{1}\right) x_{2}^{2}(t) \\
& +\max \left\{a_{3}, b_{3}\right\}\left[x_{1}^{2}(t-\tau(t))+x_{2}^{2}(t-\tau(t))\right] \\
& \leq \max \left\{-2 a_{1}+a_{2}+a_{3}+b_{1},-2 b_{2}+a_{2}+b_{3}+b_{1}\right\}\left(x_{1}^{2}(t)+x_{2}^{2}(t)\right) \\
& +\max \left\{a_{3}, b_{3}\right\} \lambda\left(x_{1}^{2}(t)+x_{2}^{2}(t)\right) \\
& =\left\{\max \left\{-2 a_{1}+a_{2}+a_{3}+b_{1},-2 b_{2}+a_{2}+b_{3}+b_{1}\right\}\right. \\
& \left.+\max \left\{a_{3}, b_{3}\right\} \lambda\right\} V(t, x(t)) \\
& =-\frac{1}{2}\left\{\min \left\{2 a_{1}-a_{2}-a_{3}-b_{1}, 2 b_{2}-a_{2}-b_{3}-b_{1}\right\}\right. \\
& \left.-\max \left\{a_{3}, b_{3}\right\}\right\} V(t, x(t)) \\
& =-\mu G(V(t, x(t))) \text {, }
\end{aligned}
$$

where $\mu=\min \left\{2 a_{1}-a_{2}-a_{3}-b_{1}, 2 b_{2}-a_{2}-b_{3}-b_{1}\right\}-\max \left\{a_{3}, b_{3}\right\}, G(s)=\frac{1}{2} s$.

Clearly, $\mu$ and $G$ satisfy the condition (iii) in Corollary 3.3.

On the other hand, we note that

$$
\begin{aligned}
V\left(t_{k}, x\left(t_{k}\right)\right)=x_{1}^{2}\left(t_{k}\right)+x_{2}^{2}\left(t_{k}\right) & =\beta_{k}^{2} x_{1}^{2}\left(t_{k}^{-}\right)+\gamma_{k}^{2} x_{2}^{2}\left(t_{k}^{-}\right) \\
& \leq\left(\max \left\{\beta_{k}, \gamma_{k}\right\}\right)^{2} V\left(t_{k}^{-}, x\left(t_{k}^{-}\right)\right)
\end{aligned}
$$

Then condition (ii) in Corollary 3.3 is satisfied. Therefore, the zero solution of (14) is uniformly stable. 
Remark 4.1. In [16], the authors obtained some conditions to guarantee the uniform stability of (14). Here we point out that the development result in Property 4.1 has wider adaptive range than that in [16]. For example, choose $a_{1}=1.75 \rho, b_{1}=0.25 \rho, a_{2}=a_{3}=b_{3}=\rho, b_{2}=2 \rho$, where $\rho>0$ is any given constant, then we get $a_{2}+a_{3}=2 \rho>a_{1}$, which implies the criteria in [16] is invalid. However, note that $\mu=0.25 \rho>0$, then we obtain that the zero solution of (4.1) with above parameters is uniformly stable.

\section{Example 4.2.}

Consider the following impulsive infinite delay differential equations:

$$
\left\{\begin{array}{l}
x^{\prime}(t)=-a(t) x(t)+b(t) \tanh (x(t-\tau))+\int_{-\infty}^{t} c(t-s) x(s) d s, t>0, t \neq t_{k}, \\
x\left(t_{k}\right)=I_{k}\left(x\left(t_{k}^{-}\right)\right), k \in \mathbb{Z}_{+}, \\
x(s)=\phi(s), s \in(-\infty, 0],
\end{array}\right.
$$

where $a, b, c \in C([0, \infty), \mathbb{R}), \tau>0,\left|I_{k}(x)\right| \leq\left(1+\beta_{k}\right)|x|, \beta_{k} \geq 0$ and $\sum \beta_{k}<\infty$.

Property 4.2. Assume that there exists constant $\lambda>1$ such that for any given $\varepsilon_{2}>\varepsilon_{1}>0$, there exists $\eta=\eta\left(\varepsilon_{1}, \varepsilon_{2}\right)>0$ such that for any $A>0$ implies that

$$
\int_{A}^{A+\eta}\left\{a(t)-\lambda\left[|b(t)|+\int_{0}^{\infty}|c(u)| d u\right]\right\} d t>\frac{2(1+\beta) \varepsilon_{2}}{\varepsilon_{1}},
$$

where $\beta=\sum \beta_{k}<\infty$.

Then the trivial solution of (16) is uniformly asymptotically stable .

Proof. In fact, let $V(t)=|x(t)|$, then it is easy to obtain Property 4.2 by Theorem 3.1. The proof procedure is repetitive and omitted here.

Remark 4.2. Here we point out that Property 4.2 can be applied to the cases not covered in $[9,11,12,19]$ even for the case of finite delay. For instance, let $a(t)=3|\sin t|, b(t)=\sin t$ and $c(t)=0$, then it is clear that all results in $[9,11,12,19]$ failed. In this case, one may choose $\lambda=2$. Then in view of the fact that

$$
\int_{\xi}^{\xi+2 \pi}|\sin t| d t>2 \text { for any constant } \xi>0
$$

For given $\varepsilon_{2}>\varepsilon_{1}>0$, we let

$$
\eta=2 \pi\left\{\left[\frac{(1+\beta) \varepsilon_{2}}{\varepsilon_{1}}\right]^{\star}+1\right\}
$$


It is easy to check that the condition in Property 4.2 is satisfied. Hence, the trivial solution of (11) with above parameters is uniformly asymptotically stable.

Remark 4.3. Note that that function $|b(t)|$ can be unbounded and the impulse constant $\mathbb{M}=$ $\prod\left(1+\beta_{k}\right)$ can be large enough. Thus our results have wider range and can be applied to some cases not covered by the results in [13-15,18-21].

\section{Conclusion}

In this paper, we further investigated the stability problem of impulsive functional differential equations with infinite delays. By using Lyapunov functions and the Razumikhin technique, some new theorems on the uniform stability and uniform asymptotic stability were obtained. Our results are milder and more general than several previously known results. But the results in this paper were only given from the impulsive perturbation point of view. How to obtain the different results, from impulsive control point of view, would be a difficult problem and need further consideration in the future.

\section{Acknowledgement}

This work was jointly supported by the Project of Shandong Province Higher Educational Science and Technology Program (J12LI04), Research Fund for Excellent Young and Middleaged Scientists of Shandong Province (BS2012DX039) and National Natural Science Foundation of China (11301308).

The research of T. Caraballo has been partially supported by FEDER (European Community) and the Spanish Ministerio de Economia y Competitividad project MTM2011-22411, and the Consejeria de Innovación, Ciencia y Empresa (Junta de Andalucia) under grant 2010/FQM314 and Proyecto de Excelencia P12-FQM-1492.

\section{References}

[1] A. Anokhin, On linear impulsive systems for functional differential equations, Soviet Mathematics Doklady 33 (1986) 220-223. 
[2] K. Gopalsamy, B. Zhang, On delay differential equations with impulses, Journal of Mathematical Analysis and Applications 139 (1989) 110-122.

[3] X. Fu, B. Yan, Y. Liu, Introduction of Impulsive Differential Systems, Science Press, Beijing, 2005.

[4] I. Stamova, G. Stamov, Impulsive control on global asymptotic stability for a class of impulsive bidirectional associative memory neural networks with distributed delays, Mathematical and Computer Modelling 53 (2011) 824-831.

[5] X. Liu, G. Ballinger, Boundedness for impulsive delay differential equations and applications to population growth models, Nonlinear Analysis 53 (2003) 1041-1062.

[6] A. Ignatyev, On the stability of invariant sets of systems with impulse effect, Nonlinear Analysis 69 (2008) 53-72.

[7] I. Stamova, G. Stamov, Stability analysis of impulsive functional systems of fractional order, Communications in Nonlinear Science and Numerical Simulation 19 (2014) 702-709.

[8] J. Shen, J. Yan, Razumikhin type stability theorems for impulsive functional differential equations, Nonlinear Analysis 33 (1998) 519-531.

[9] F. Chen, X. Wen, Asymptotic stability for impulsive functional differential equation, Journal of Mathematical Analysis and Applications 336 (2007) 1149-1160.

[10] S. Zavalishchin, A. Sesekin, Dynamic Impulse Systems Theory and Applications, Kluwer Academic Publishers Group, Dordrecht, 1997.

[11] Q. Wang, X. Liu, Exponential stability for impulsive delay differential equations by Razumikhin method, Journal of Mathematical Analysis and Applications 309 (2005) 462-473.

[12] B. Liu, X. Liu, K. Teo, Q. Wang, Razumikhin-type theorems on exponential stability of impulsive delay systems, IMA Journal of Applied Mathematics 71 (2006) 47-61.

[13] Z. Luo, J. Shen, Stability of impulsive functional differential equations via the Liapunov functional, Applied Mathematics Letters 22 (2009) 163-169.

[14] Z. Luo, J. Shen, Stability results for impulsive functional differential equations with infinite delays, Journal of Computational and Applied Mathematics 131 (2001) 55-64.

[15] Z. Luo, J. Shen, Stability and boundedness for impulsive functional differential equations with infinite delays, Nonlinear Analysis 46 (2001) 475-493.

[16] Y. Zhang, J. Sun, Stability of impulsive infinite delay differential equations, Applied Mathematics Letters 19 (2006) 1100-1106.

[17] S. Zhang, A new technique in stability of infinite delay differential equations, Computers Mathematics with Applications 44 (2002) 1275-1287.

[18] T. Faria, M. Gadotti, J. Oliveira, Stability results for impulsive functional differential equations with infinite delay, Nonlinear Analysis: Theory, Methods and Applications 75 (2012) 6570-6587.

[19] X. Fu, X. Li, Razumikhin-type theorems on exponential stability of impulsive infinite delay differential systems, Journal of Computational and Applied Mathematics 224 (2009) 1-10.

[20] X. Li, New results on global exponential stabilization of impulsive functional differential equations with 
infinite delays or finite delays, Nonlinear Analysis Series B: Real World Applications 11 (2010) 4194-4201.

[21] X. Li, H. Akca, X. Fu, Uniform stability of impulsive infinite delay differential equations with applications to systems with integral impulsive conditions, Applied Mathematics and Computation 219 (2013) 73297337. 